SLAC-PUB-8340

January 2000

\title{
Soft Colour Interactions in Non-perturbative QCD
}

\author{
G. Ingelman et al.
}

Contributed to 15th International Conference on Particle and Nuclei (PANIC 99), 6/10/1999—6/16/1999, Uppsala, Sweden

Stanford Linear Accelerator Center, Stanford University, Stanford, CA 94309

Work supported by Department of Energy contract DE-AC03-76SF00515. 
TSL/ISV-99-0222

October 1999

\title{
Soft Colour Interactions in Non-perturbative QCD*
}

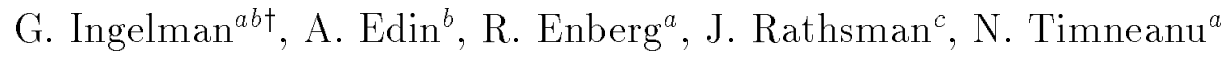 \\ ${ }^{a}$ Dept. of Radiation Sciences, Uppsala University, Box 535, S-751 21 Uppsala, Sweden \\ ${ }^{b}$ DESY, Notkestrasse 85, D-22603 Hamburg, Germany \\ ${ }^{c}$ SLAC, Stanford, California 94309, USA
}

Improved understanding of non-perturbative QCD dynamics can be obtained in terms of soft colour exchange models. Their essence is the variation of colour string-field topologies giving a unified description of final states in high energy interactions. In particular, both events with and without large rapidity gaps are obtained in agreement with data from $e p$ at HERA and $p \bar{p}$ at the Tevatron, where also the surprisingly large production rate of high- $p_{\perp}$ charmonium and bottomonium is reproduced.

Strong interaction processes at small ('soft') momentum transfers belong to the realm of non-perturbative QCD, which is a major unsolved problem in particle and nuclear physics. High energy particle collisions involving a 'hard' scale, i.e. a large momentum transfer, has the advantage of providing a well defined parton level process which is calculable in perturbative QCD (pQCD). The soft effects (e.g. hadronisation) in such hard scattering events can therefore be investigated based on an understood parton level process.

This hard-soft interplay is the basis for the topical research field of diffractive hard

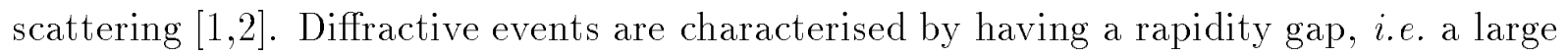
region of rapidity (or polar angle) without any particles. The rapidity gap connects to the soft part of the event and therefore non-perturbative effects on a long space-time scale are important.

In order to better understand non-perturbative dynamics and provide a unified description of all final states, we have developed new models. These models are added to Monte

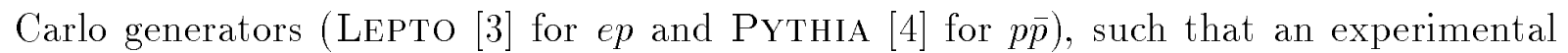
approach can be taken to classify events depending on the characteristics of the final state: e.g. gaps or no-gaps, leading protons or neutrons etc.

The basic assumption of the models is that variations in the topology of the confining

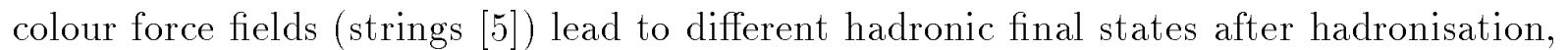
as illustrated in Figs. ${ }_{-1}^{1}$ and colour order. However, this order may change due to soft, non-perturbative interactions.

*Contribution to PANIC 99 conference proceedings

$\dagger$ ingelman@tsl.uu.se www3.tsl.uu.se/thep/ 

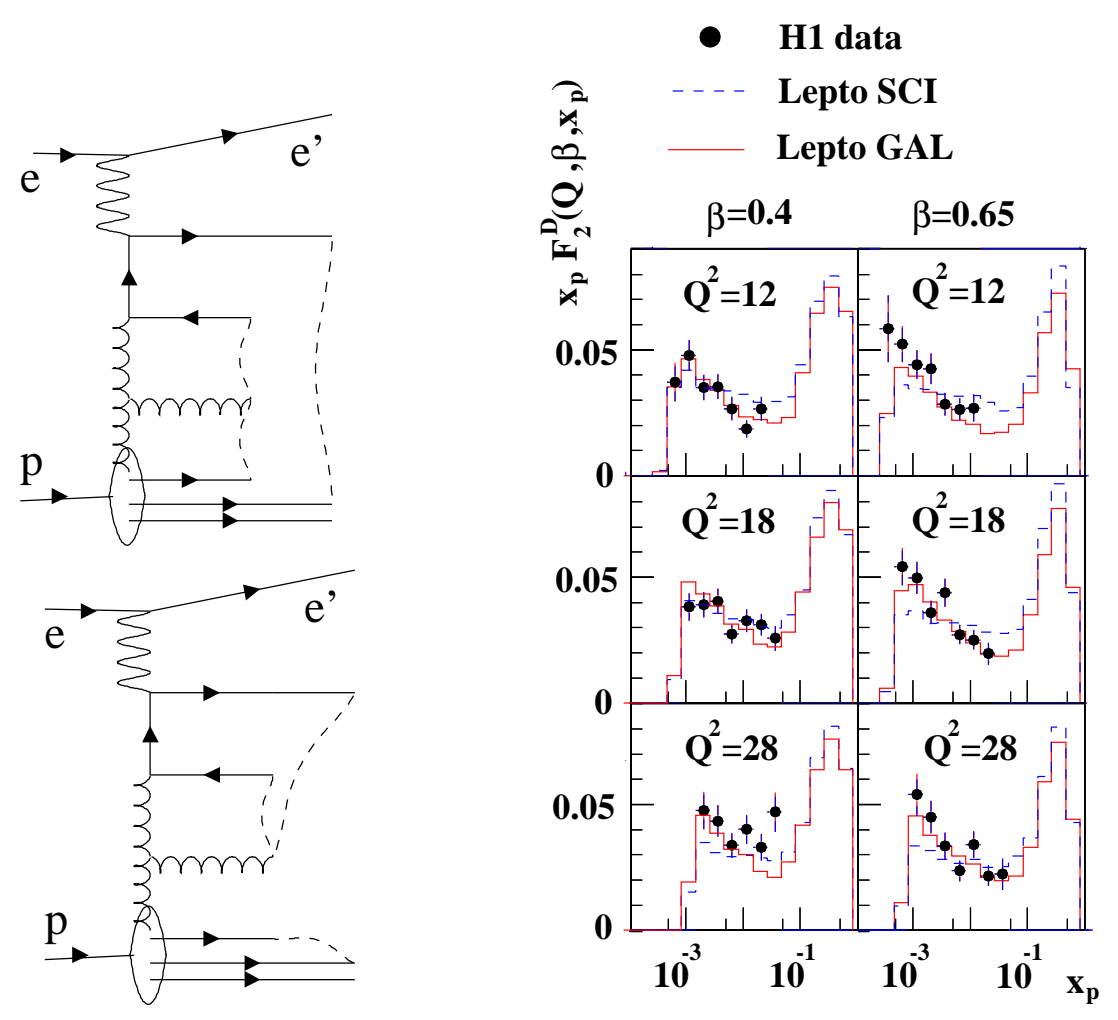

Figure 1. Gluon-induced deep inelastic scattering event with examples of colour string connection (dashed lines) of partons in conventional Lund model [is] based on the colour order in pQCD (upper left), and after colour rearrangement through SCI or GAL mechanisms (lower left). The diffractive structure function $F_{2}^{D(3)}\left(x_{\mathbb{P}} ; \beta, Q^{2}\right)$ in a selected kinematic range with $\mathrm{H} 1$ data [is] compared to the SCI and GAL models; excerpt from [i].].

In the soft colour interaction (SCI) model [6] it is assumed that colour-anticolour, corresponding to non-perturbative gluons, can be exchanged between partons and remnants emerging from a hard scattering. This can be viewed as the partons interacting softly with the colour medium of the proton as they propagate through it, which should be a natural part of the process in which 'bare' perturbative partons are 'dressed' into non-perturbative ones and the confining colour flux tube between them is formed. The hard parton level interactions are given by standard perturbative matrix elements and parton showers, which are not altered by softer non-perturbative effects. The unknown probability to exchange a soft gluon between parton pairs is given by a phenomenological parameter $R$, which is the only free parameter of the model. With $R=0.5$ one obtains [i] the correct rate of rapidity gap events observed at HERA and a quite decent description of the measured diffractive structure function (Fig. 留).

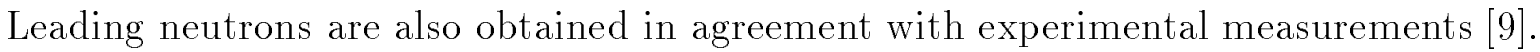
In the Regge approach pomeron exchange would be used for diffraction, pion exchange added to get leading neutrons and still other exchanges should be added for completeness. The SCI model provides a simpler description.

Applying the same SCI model to hard $p \bar{p}$ collisions one obtains production of $W$ and 

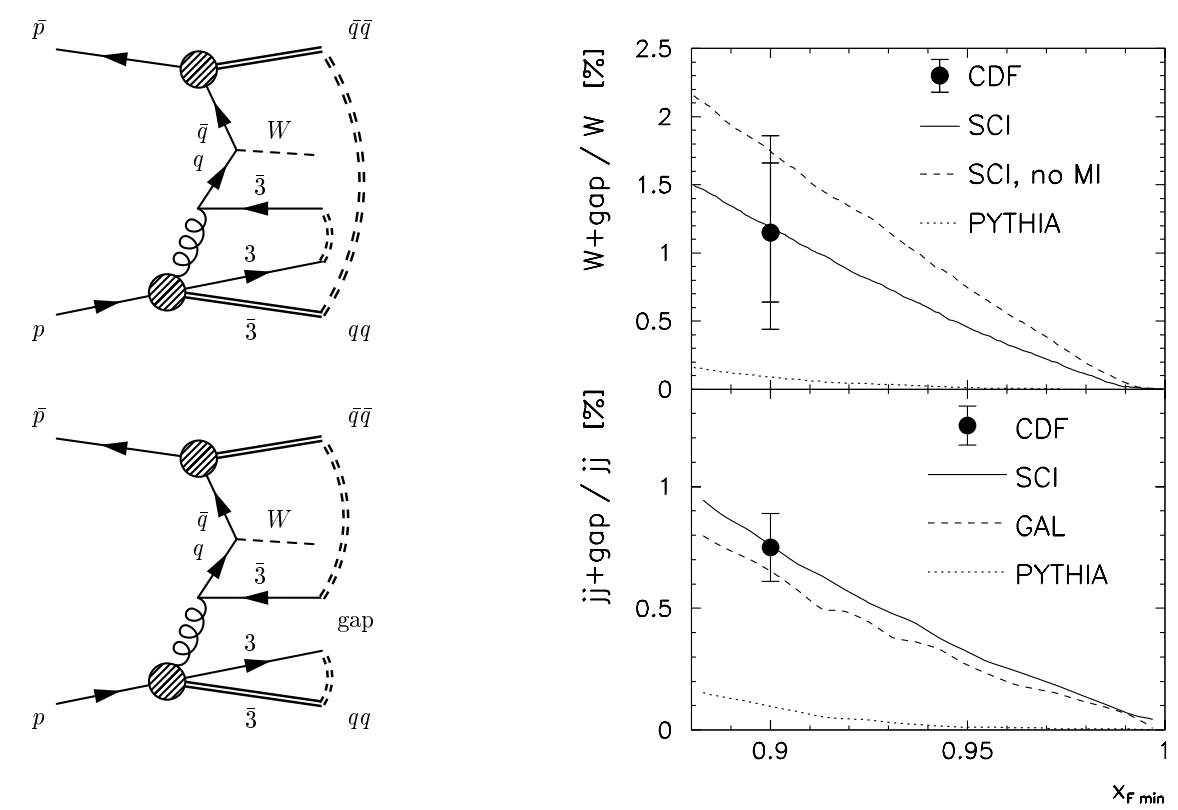

Figure 2. $W$ production in $p \bar{p}$ with string topology before and after colour rearrangement resulting in a gap. Relative rate of $W$ and two-jet $(j j)$ events with a rapidity gap corresponding to diffraction with a leading proton with minimum $x_{F}=0.9$ in Tevatron data [1] and in the SCI and GAL models. Sensitivity to multiparton interactions (MI) and results from standard PYTHIA without soft colour exchanges is also shown [i] $\overline{0}]$.

jets in association with rapidity gaps. As shown in Fig. observed at the Tevatron using the same $R$-value as obtained from gaps at HERA. This is in contrast to the Pomeron model which, when tuned to HERA gap events, gives a factor

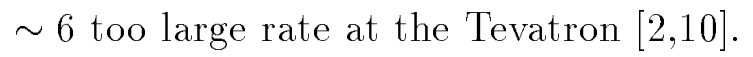

SCI does not only lead to rapidity gaps, but also to other striking effects. It reproduces (Fig. ${ }_{-1}^{13}$ ) the observed rate of high- $p_{\perp}$ charmonium and bottomonium at the Tevatron, which are factors of 10 larger than predictions based on conventional pQCD. This is accomplished by the change of the colour charge of a $Q \bar{Q}$ pair (e.g. from a gluon) from octet to singlet. A quarkonium state can then be formed using a simple model for the division of the cross-section below the threshold for open heavy flavour production onto different quarkonium states [i] $\overline{2}$ ].

An alternative to SCI is the newly developed generalised area law (GAL) model [i] which, based on a generalisation of the area law suppression $e^{-b A}$ with $A$ the area swept out by the string in energy-momentum space, gives modified colour string topologies through string reinteractions. The probability $P=R_{0}[1-\exp (-b \Delta A)]$ for two strings pieces to interact depends on the area difference $\Delta A$ which is gained by the string rearrangement. This favours making 'shorter' strings, e.g. with gaps, whereas making 'longer', 'zig-zag' shaped strings is suppressed. The fixed probability $R$ in SCI is thus replaced by a dynamical one, where the parameter $R_{0}=0.1$ is chosen to reproduce the HERA gap event rate in a simultaneous fit to data from $e^{+} e^{-}$annihilation at the $Z^{0}$-peak. The resulting 


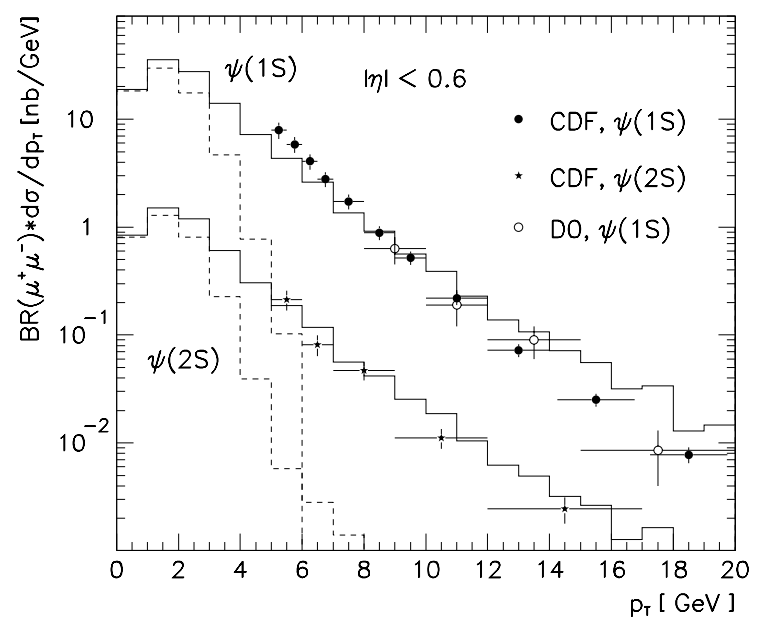

Figure 3. Transverse momentum spectra of prompt charmonium $\left(J / \psi\right.$ and $\left.\psi^{\prime}\right)$ in $p \bar{p}$ collisions at the Tevatron energy. Data from CDF and D0 compared to results from the SCI model (histograms) applied to $c \bar{c}$ production from leading order $\left(\alpha_{s}^{2}\right)$ matrix elements (dashed lines) and with inclusion of higher order contributions calculated in the parton shower approach (full lines). From [i] 2 .

diffractive structure function compares very well with HERA data (Fig. 咞). The GAL model also improves the description of non-diffractive HERA data [it].

The GAL model can also be applied to $p \bar{p}$ to obtain diffractive $W$ and jet production through string rearrangements like in Fig. ${ }_{i}^{2}$ The observed rates are reproduced quite well (Fig. 2i-1). However, the treatment of the 'underlying event', which is a notorious problem in hadron-hadron scattering, introduces a larger uncertainty than for the SCI model [i] $\left.{ }_{1}^{\overline{1}} \overline{0}\right]$.

In conclusion, our models for non-perturbative QCD dynamics in terms of varying colour string topologies give a satisfactory unified description of several phenomena in different hadronic final states. This should contribute to a better understanding of nonperturbative QCD interactions.

\section{REFERENCES}

1. G. Ingelman, P.E. Schlein, Phys. Lett. B152 (1985) 256

2. G. Ingelman, 'Diffractive hard scattering', DESY 99-009, in proc. Techniques and Concepts of High Energy Physics, Ed. T. Ferbel, Kluwer academic publishers 1999

3. G. Ingelman, A. Edin, J. Rathsman, Comput. Phys. Commun. 101 (1997) 108

4. T. Sjöstrand, Comput. Phys. Commun. 82 (1994) 74

5. B. Andersson, G. Gustafson, G. Ingelman, T. Sjöstrand, Phys. Rep. 97 (1983) 31

6. A. Edin, G. Ingelman, J. Rathsman, Phys.Lett. B366 (1996) 371; Z.Phys. C75 (1997) 57

7. A. Edin, G. Ingelman, J. Rathsman, TSL/ISV-99-0215, in proc. 'Monte Carlo generators for HERA physics', DESY-PROC-1999-02 p. 280, www.desy.de/ heramc

8. C. Adloff et al. (H1), Z. Phys. C76 (1997) 613

9. C. Adloff et al., Eur. Phys. J. C6 (1999) 587

10. R. Enberg, G. Ingelman, N. Timneanu, in preparation

11. F. Abe et al.(CDF), Phys.Rev.Lett. 78 (1997) 2698; Phys. Rev. Lett. 79 (1997) 2636

12. A. Edin, G. Ingelman, J. Rathsman, Phys. Rev. D56 (1997) 7317

13. J. Rathsman, Phys. Lett. B452 (1999) 364 